\title{
Bilateral vocal fold injection with autologous fat in patients with vocal fold atrophy with or without sulcus
}

\author{
Emke M. J. M. van den Broek ${ }^{1,2}$ (D) Bas J. Heijnen ${ }^{1} \cdot$ Martine Hendriksma $^{1} \cdot$ Vivienne A. H. van de Kamp-Lam ${ }^{1}$. \\ Antonius P. M. Langeveld ${ }^{1}$. Peter Paul G. van Benthem ${ }^{1}$ • Elisabeth V. Sjögren ${ }^{1}$
}

Received: 29 March 2019 / Accepted: 16 May 2019 / Published online: 27 May 2019

(c) The Author(s) 2019

\begin{abstract}
Purpose To evaluate voice outcome after bilateral vocal fold injection with autologous fat in patients with non-paralytic glottic insufficiency due to vocal fold atrophy with or without sulcus.

Methods This is a retrospective cohort study from September 2012 to December 2017 including 23 patients undergoing bilateral vocal fold injection with autologous fat ( 24 procedures) for vocal fold atrophy (15 procedures) or atrophy with sulcus (Ford type II or III) (9 procedures). Voice data were collected and analyzed for the preoperative and the 3- and 12-month postoperative time points according to a standardized protocol, including Voice Handicap Index (VHI)-30 and perceptive, acoustic and aerodynamic parameters. Failure rate was defined as non-relevant improvement $(<10$ points $)$ in VHI-30 at 12 months and number of revisions within 12 months.

Results There was a clinically relevant ( $\geq 15$ points) and statistically significant improvement in the VHI-30 (preoperative: 49.1 points; postoperative at 12 months: 29.7 points). Change in dynamic range was also statistically significant over time $(p=0.028)$. There were no differences in voice parameters between patients with atrophy only and atrophy with sulcus, although grade tended to be lower in patients with atrophy only over all time points.

Conclusion This study shows that bilateral vocal fold injection with autologous fat is a beneficial treatment not only for patients with atrophy but also for patients with sulcus. A comparison of the results with those reported from other forms of sulcus surgery confirmed this finding. However, there is a need for further prospective studies comparing the short- and long-term effects of different techniques.
\end{abstract}

Keywords Glottic insufficiency $\cdot$ Vocal fold atrophy $\cdot$ Sulcus $\cdot$ Autologous fat $\cdot$ Vocal fold injection

\section{Introduction}

Non-paralytic glottic insufficiency is a common cause of dysphonia. There are several underlying causes, including vocal fold atrophy. In our clinic, we routinely encounter three forms of vocal fold atrophy: vocal fold atrophy in presbyphonia, an adolescent form, and atrophy associated with congenital vocal fold scar in the form of sulcus [1]. If a sulcus is present it can be further classified as a physiologic

Emke M. J. M. van den Broek

E.M.J.M.van_den_Broek@lumc.nl

1 Department of Otorhinolaryngology/Head and Neck Surgery, Leiden University Medical Centre, Albinusdreef 2, PO-box 9600, 2300 RC Leiden, The Netherlands

2 Department of Otorhinolaryngology/Head and Neck Surgery, University Medical Centre, Utrecht, The Netherlands sulcus (Ford type I) or pathologic sulcus vocalis (Ford types II and III) with Ford types II and III corresponding to a sulcus vergeture and a sulcus vocalis in the classification by Bouchayer and Cornut [2,3].

The main surgical treatment for atrophy without sulcus is vocal fold medialization, which can be achieved either by bilateral vocal fold injection (VFI) with a durable injectable such as autologous fat or calcium hydroxyapatite, or by bilateral medialization thyroplasty. For vocal fold atrophy with sulcus, several surgical techniques are used that are broadly divided into phonosurgical epithelium freeing techniques such as microflap formation, hydrodissection, angiolytic laser treatment and tissue engineering techniques on the one hand, and medialization techniques on the other. In their consensus report on vocal fold scar, the European Laryngological Society (ELS) considered medialization to be the least traumatizing procedure to the vocal fold and, 
therefore, suggested that it be used as the initial treatment for vocal fold scar, including sulcus [4]. However, it is also known that the results of medialization for vocal fold atrophy with scar, including sulcus, are less predictable than the results for glottic insufficiencies caused by atrophy alone, hypomobility, or paresis [5]. In this study, we evaluated the prospectively collected voice outcome data after bilateral VFI with autologous fat in patients with vocal fold atrophy with or without sulcus and compared our findings with those reported in the literature.

\section{Methods}

\section{Patients}

This study was approved by the Leiden University Medical Centre Ethics Committee. All patients with non-paralytic glottic insufficiency who underwent bilateral VFI with autologous fat under general anesthesia $(n=32$, procedures $=35)$ from September 2011 to December 2017 were retrospectively reviewed. Seven patients were excluded because of previous phonosurgery for sulcus $(n=1)$, paresis as another cause of glottic insufficiency $(n=2)$, or an underlying disease affecting the voice $(n=4)$ including Parkinson's disease $(n=2)$, laryngeal dystonia $(n=1)$, and laryngeal papillomatosis $(n=1)$. Of the 25 remaining patients ( 28 procedures), 23 ( 24 procedures) had pre- and postoperative voice data with at least a complete Voice Handicap Index (VHI)-30 questionnaire and were included in the definitive analysis (Fig. 1). These patients had undergone bilateral VFI with autologous fat between September 2012 and December 2017.

\section{Voice data}

Voice outcome data were collected according to a standardized voice analysis protocol implemented preoperatively and at 3 and 12 months postoperatively. This protocol included patients' self-assessments using the VHI-30, perceptual evaluation using the overall grade score of the GRBAS (Grade, Roughness, Breathiness, Asthenia, Strain) scale, aerodynamic evaluation with maximum phonation time (MPT) and dynamic range, and acoustic analyses including fundamental frequency (F0) and melodic range. The VHI-30 was the primary outcome parameter of the voice analysis protocol. It is a patient-based self-assessment tool consisting of 30 items, which are distributed over three domains: functional, physical, and emotional [6]. In the Dutch version of the VHI-30, a score of 15 points or more identifies patients with voice problems in daily life $[7,8]$. Furthermore, a change in pre- and postoperative score of 10 points or more in the individual patient and 15 points or more for a group can be considered clinically relevant [8]. The voice was perceptually graded using the grade of the GRBAS scale ranging from zero to three [9]. Running speech samples in random order were graded by experienced listeners (two senior speech language therapists and one laryngologist) and a consensus was reached through (re)evaluation and discussion. The MPT was measured on /a/ at a comfortable pitch and loudness. The longest MPT from two attempts was included in the analysis. The fundamental frequency in hertz $(\mathrm{Hz})$, dynamic range in decibels $(\mathrm{dB})$, and melodic range in semitones (ST) were extracted from the patient's phonetogram recorded with the voice profiler (Alphatron, Rotterdam, the Netherlands, 2007) using standardized settings.
Fig. 1 Patient selection and inclusion and exclusion criteria

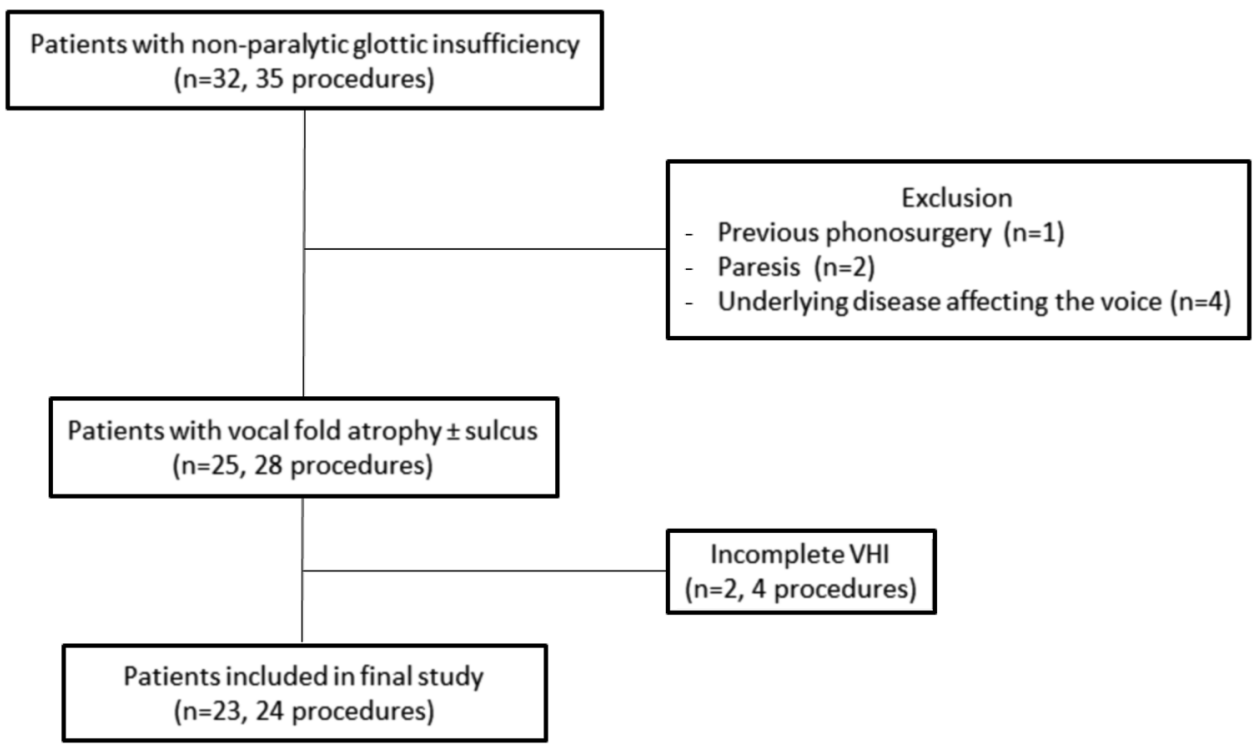




\section{Procedure}

All procedures were performed under general anesthesia by an experienced laryngologist and/or a fellowship-trained laryngologist. Bilateral VFI was performed with autologous fat, harvested by abdominal liposuction. In a minority of patients using anticoagulants, a periumbilical incision was made to ensure hemostasis, and fat lobules were harvested and separated from the underlying connective tissue. Subsequently, the fat was centrifuged and separated from blood and its liquid component. The standard practice was injection of fat lateral in the vocal fold (thyroarytenoid muscle) at a level just anterior of the vocal fold process using a Brünings syringe until medialization was achieved, with an anticipated overcorrection of between $1 / 3$ and $1 / 4$ of the vocal fold width at the free edge. The final amount on overcorrection was based on the clinical experience of the surgeon. In some cases, a second injection point at the level of the midcord was needed to obtain the intended result. All patients had absolute voice rest for $24 \mathrm{~h}$ after the procedure. Subsequently, they received voice therapy by an experienced speech-language therapist, starting within the first week postoperatively, including resonant voice therapy and vocal hygiene advice.

\section{Statistical analysis}

All data were analyzed using SPSS (IBM SPSS Statistics for Windows, Version 21.0, released 2012. IBM Corp, Armonk, NY, USA). Demographic details were presented as the mean with standard deviation (SD) or as proportions using percentages. The effect of time on the different voice parameters was assessed with the linear mixed model and was adjusted for diagnosis (atrophy versus atrophy with sulcus). The linear mixed model was chosen because it applies a correction for missing data. This correction is based on the observed data and uses all available data, without the need to censure a patients' entire data, when one or more data points are missing or the need for imputation of measurements [10]. For all statistical tests, a $p$ value $<0.05$ was considered significant.

\section{Results}

Table 1 shows the preoperative demographic details of the 23 patients undergoing 24 procedures. These were all female patients. Fifteen injections were performed in patients with vocal fold atrophy, two of these were performed consecutively in the same patient within 5 months. Nine injections were performed in patients with atrophy and sulcus (Ford type II or III). The pre- and post-VFI voice outcome data for the overall patient group are
Table 1 Demographic details of the patients undergoing bilateral vocal fold injection with autologous fat

\begin{tabular}{ll}
\hline Characteristics & Total=24 (100\%) \\
\hline $\begin{array}{l}\text { Mean age, years at baseline (SD) } \\
\text { Gender (\%) }\end{array}$ & $39.5(18.2)$ \\
Female & $24(100)$ \\
Etiology (\%) & \\
Atrophy & $15(62.5)$ \\
Atrophy with sulcus & $9(37.5)$ \\
\hline
\end{tabular}

$S D$ standard deviation

shown in Table 2 . The changes in VHI-30 $(p<0.001)$ and dynamic range $(p=0.028)$ were statistically significant over time. The change in the VHI-30 from baseline to 12 months postoperation was also clinically relevant $(\Delta$ 19.4). The improvement in postoperative outcomes in the other voice parameters was not statistically significant (Table 2).

Table 3 shows the results stratified for patients with vocal fold atrophy only and patients with vocal fold atrophy and sulcus. The overall change in the VHI-30 was statistically significant in both groups $(p<0.001, p=0.002)$. The change in the VHI from the preoperative time point to the 12-month postoperative time point was clinically relevant in both groups ( $\Delta 21.2$ atrophy; $\Delta 17.7$ sulcus). There was no significant improvement in any of the other voice parameters for the two groups. Finally, there was no significant difference between the two groups in the severity of the scores for the various voice parameters, although the grade of dysphonia tended to be lower in patients with atrophy (mean difference over all time points $0.47, p=0.057$, data not shown in table).

Looking at individual procedures, 12 -month data were available in 18 out of 24 procedures. Six procedures did not have 12-month data: in four procedures, the patients $(n=4)$ had already undergone or opted for a revision procedure [repeat VFI with fat $(n=2)$ or medialization thyroplasty $(n=2)]$ and in two procedures the patients $(n=2)$ were lost to follow-up. Out of 18 procedures VHI-improvement at 12 months could be calculated; 15 procedures had a clinically relevant improvement $(\geq 10$ points) in the VHI-30. Three out of 18 had a non-relevant improvement $(<10$ points). We, therefore, estimate the failure rate within 12 months for individual procedures to be somewhere between $16.7 \%$ (4/24 procedures) if considering only procedures requiring revision as failures and $37.5 \%$ (9/24 procedures) if considering procedures requiring revision and procedures without clinically relevant VHI improvement as failures and hypothesizing that the two procedures lost to follow-up were also failures. 
Table 2 Pre- and postoperative voice outcome data of patients with vocal fold atrophy \pm sulcus undergoing bilateral vocal fold injection with autologous fat
Table 3 Pre- and postoperative voice outcome data stratified for patients with vocal fold atrophy and vocal fold atrophy with sulcus

\begin{tabular}{llllc}
\hline & $\begin{array}{l}\text { Preoperative } \\
\text { Mean }(95 \% \mathrm{CI})\end{array}$ & $\begin{array}{l}3 \text { months postoperation } \\
\text { Mean }(95 \% \mathrm{CI})\end{array}$ & $\begin{array}{l}12 \text { months postoperation } \\
\text { Mean }(95 \% \mathrm{CI})\end{array}$ & $p$ value \\
\hline VHI-30 & $49.1(41.4 ; 56.8)$ & $34.8(27.0 ; 42.6)$ & $29.7(21.5 ; 37.9)$ & $<0.001^{*}$ \\
Grade & $1.58(1.3 ; 1.9)$ & $1.20(0.9 ; 1.5)$ & $1.18(0.8 ; 1.6)$ & 0.071 \\
MPT (s) & $11.0(9.2 ; 12.9)$ & $12.4(10.5 ; 14.3)$ & $12.2(10.0 ; 14.4)$ & 0.384 \\
Dynamic range (dB) & $32.3(27.5 ; 37.0)$ & $33.8(29.1 ; 38.5)$ & $41.1(35.4 ; 46.8)$ & $0.028^{*}$ \\
F0 (Hz) & $198(187 ; 209)$ & $201(190 ; 212)$ & $196(184 ; 209)$ & 0.730 \\
Melodic range (ST) & $17.0(14.1 ; 19.8)$ & $17.4(14.5 ; 20.3)$ & $19.7(16.2 ; 23.2)$ & 0.428 \\
\hline
\end{tabular}

$C I$ confidence interval, $V H I$ Voice Handicap Index, $M P T$ maximum phonation time, $F O$ fundamental frequency, $H z$ hertz, $d B$ decibels, $S T$ semitones

* $p$ value $<0.05$ was considered significant

\begin{tabular}{lllllc}
\hline & Etiology & $\begin{array}{l}\text { Preoperative } \\
\text { Mean }\end{array}$ & $\begin{array}{l}\text { 3 months post- } \\
\text { operation } \\
\text { Mean }\end{array}$ & $\begin{array}{l}\text { 12 months post- } \\
\text { operation } \\
\text { Mean }\end{array}$ & $p$ value \\
\hline VHI-30 & Atrophy & 47.5 & 33.0 & 26.3 & $<0.001^{*}$ \\
& Sulcus & 51.8 & 38.0 & 34.1 & $0.002^{*}$ \\
Grade & Atrophy & 1.5 & 0.9 & 1.0 & 0.078 \\
& Sulcus & 1.8 & 1.6 & 1.5 & 0.602 \\
MPT (s) & Atrophy & 11.2 & 13.4 & 12.4 & 0.292 \\
& Sulcus & 10.7 & 10.9 & 11.8 & 0.825 \\
Dynamic range (dB) & Atrophy & 30.6 & 33.3 & 41.6 & 0.051 \\
& Sulcus & 35.0 & 34.6 & 40.6 & 0.449 \\
F0 (Hz) & Atrophy & 191 & 199 & 194 & 0.513 \\
& Sulcus & 210 & 205 & 200 & 0.642 \\
Melodic range (ST) & Atrophy & 15.7 & 17.5 & 19.8 & 0.363 \\
& Sulcus & 19.1 & 17.2 & 19.6 & 0.743 \\
\hline
\end{tabular}

$V H I$ Voice Handicap Index, $M P T$ maximum phonation time, $F O$ fundamental frequency, $H z$ hertz, $d B$ decibels, $S T$ semitones

$p$ value $<0.05$ was considered significant

\section{Discussion}

In this study, we evaluated the voice outcome data after bilateral VFI of autologous fat in patients with vocal fold atrophy with or without sulcus. We found a statistically significant and clinically relevant improvement in the VHI-30 for the study group as a whole. This improvement was consistent in both patient groups (atrophy alone and atrophy with sulcus). Therefore, our results show that bilateral VFI with autologous fat is a beneficial treatment for patients with vocal fold atrophy with or without sulcus. Additionally, our results suggest that it is equally beneficial in both these patient groups. Although our results demonstrated voice improvement, there was no normalization of the voice and/or voice use based on the 12-month postoperative VHI-30 score remaining above 15 points, which indicates an elevated patient-perceived voicerelated handicap.
The improvement in the VHI is supported by a statistically significant improvement in the dynamic range. Although there are no clear definitions as to what constitutes a clinically meaningful improvement in this parameter, we believe that this improvement in volume range from 32.3 to $41.1 \mathrm{~dB}$ is most likely meaningful to the patient, taking into account that range of $46 \mathrm{~dB}$ is considered acceptable for a normal voice [11]. Functionally, this reflects the likelihood that patients will notice that less effort is required to produce a softer or louder voice. Effort, and not the quality of the voice, is often the main complaint of patients with atrophy with or without sulcus and the primary aim when performing these procedures is most often to improve their voice function.

Several studies have shown a beneficial effect of autologous fat injection on glottic insufficiency from both paralytic and non-paralytic causes [12-20]. Autologous fat injection is known to be safe [21], but the long-term ( $>1$ year) benefits are less consistent $[12,19,22,23]$. The effectiveness 
of autologous fat injection in patients with non-paralytic glottic insufficiency caused by atrophy with or without sulcus has been studied to a lesser extent, with generally positive subjective results [19, 22-24]. Chen et al. reported excellent results in $62.5 \%$ of their patients based on subjective patient ratings, together with an overall significant improvement in the perceptual rating of grade, roughness, and breathiness, and videolaryngostroboscopic rating [22]. Although Chen and others suggest that the results of fat injection are better in patients with atrophy alone than atrophy with sulcus $[14,22]$, this was not confirmed in our study. Recently, Dominguez et al. reported good results for patients with vocal atrophy and/or paresis treated with fat injection or bilateral medialization thyroplasty [19]. However, they found that only the thyroplasty group maintained this effect during the whole follow-up period (19 months for fat injection, 16.3 months for thyroplasty). Although the reabsorption of fat is a well-acknowledged downside of the procedure, which we anticipated, we did not see this when looking at our overall VHI-30 scores at 12 months. However, failure rate in the first 12 postoperative months in this study was estimated to be between 17 and $37.5 \%$. A longer followup is required to more definitely establish the life span of fat injections in our population. Possible explanations for the discrepancies in the literature on this topic can be the amount of overcorrection or the technique of fat preservation used and the timing of post-operative measurements. It is important to establish firmly if long-term results do indeed favor thyroplasty over VFI as medialization technique.

To determine the optimal treatment for sulcus, we compared our results from bilateral VFI with autologous fat with those from other forms of sulcus surgery. The main alterative is microphonosurgery to free the epithelium and many different techniques have been described, mostly retrospectively and with varying effects.

Stuut et al. found no improvement in VHI (pre-operative 48.8, postoperative 47.1) in their patients with sulcus glottidis $(n=17)$ using solely the epithelium-freeing technique as described by Bouchayer [25]. Therefore, it has been suggested by them and by others to not only dissect the sulcus, but to restore the layered structure of the vocal folds to improve treatment outcome. Positive results have been achieved using transplantation of autologous temporalis fascia into the vocal fold (ATFV). In a study showing long-term results of ATFV in 21 patients, the VHI-10 decreased by 8.35 points after 6 months and 13.53 points after 44 months. These improvements were both statistically $(p<0.001)$ and clinically relevant $(\geq 5$ points on the VHI-10 [26]) [27]. However, the mean VHI-10 at last follow-up was still just above 11 points, which is considered abnormal [26]. A recent cohort of ten patients with sulcus vocalis $(n=6)$ and vocal fold scar $(n=4)$ confirmed the beneficial effect of ATFV with significant improvements in the VHI-10, perceptual grading, as well as aerodynamic (MPT, $s / z$ ratio) and stroboscopic findings 6 months after the operation [28]. There are also studies that combine the excision of the sulcus with medialization of the vocal fold [29-31]. Yilmaz reported on 44 patients with sulcus who underwent excising of the sulcus followed by injection medialization with hyaluronic acid or calcium hydroxyapatite $(n=42)$ or bilateral type 1 thyroplasty $(n=2)$. The VHI-30 improved significantly (90.5-39.1 points) at a mean follow-up of 30 months. There was also significant improvement in the grade, roughness and breathiness of the GRBAS score, glottal closure and mucosal wave amplitude of the stroboscopic findings, and in most of the aerodynamic and acoustic parameters [29]. Miaskiewicz recently showed an improvement in the VHI-30 of 10.2 points (preoperative 44.36 versus postoperative 34.12) at 12 months after combined sulcus excision and VFI, using a short-acting filler in 29 cases, a long-acting filler in 2 , a combination of fillers in 2 , and no filler in 3 cases [30].

The use of angiolytic lasers for sulcus vocalis is a relatively new treatment option conceptually based on using selective photothermolysis to soften scar tissue [32-34]. A recently published series of 79 patients showed an improvement in the VHI-30 of 19.76 points, to a score close to 40 points after 6 months, as well as a significant improvement in the GRBAS by 1.07 points. Several objective voice parameters (noise to harmonic ratio, jitter, shimmer, MPT) also improved significantly in this study [34].

Despite their different surgical approaches to treat sulcus, the studies above show statistically significant and clinically relevant improvements in VHI scores, although these are still above normal [27, 29, 30, 34]. It is important to note that these results are in line with our results from treating sulcus with autologous fat injection only. Therefore, at the moment these techniques can be considered equal as to short-term (12 months) treatment effect. This is supported by the findings of Welham et al. in their prospective trial comparing three types of surgery for sulcus: type I thyroplasty, VFI with synthetic filler, and graft implantation. They concluded that no single treatment option is completely successful and there is no evidence-based decision algorithm available to identify the optimal treatment for an individual patient with sulcus [35]. Therefore, at this stage, our findings support the recommendation in the ELS consensus report to start with the least invasive technique for sulcus [4], i.e., VFI. We emphasize the importance of larger prospective studies comparing the different surgical techniques to establish optimal treatment algorithms for these patients.

Our study has some limitations. Our patient population was relatively small, although it was one of the larger study cohorts on this subject. We advocate a prospective study with a larger cohort. Also, our follow-up at this time was limited to 12 months. As stated above, a longer follow-up 
would better establish the life span of fat injections in our population.

\section{Conclusion}

This study shows a statistically significant and clinically relevant subjective improvement up to 12 months after VFI with autologous fat in patients with vocal fold atrophy with or without sulcus. This improvement was reflected in the dynamic capabilities of the patients' voices. The degree of improvement was similar in patients with or without sulcus. This indicates that bilateral VFI with autologous fat is a beneficial treatment option not only for patients with atrophy but also for patients with sulcus. Comparing our results with those from other forms of sulcus surgery in the literature confirms this finding; although we emphasize the need for further prospective studies comparing the short- and longterm effects of different techniques.

\section{Compliance with ethical standards}

Conflict of interest The authors declare that they have no conflict of interest.

Open Access This article is distributed under the terms of the Creative Commons Attribution 4.0 International License (http://creativeco mmons.org/licenses/by/4.0/), which permits unrestricted use, distribution, and reproduction in any medium, provided you give appropriate credit to the original author(s) and the source, provide a link to the Creative Commons license, and indicate if changes were made.

\section{References}

1. Van den Broek EMJM, Heijnen BJ, Hendriksma M, Langeveld APM, van Benthem PPG, Sjögren EV (2019) Bilateral trial vocal fold injection with hyaluronic acid in patients with vocal fold atrophy with or without sulcus. Eur Arch Otorhinolaryngol 276(5):1413-1422. https://doi.org/10.1007/s00405-019-05347-2

2. Ford CN, Inagi K, Khidr A, Bless DM, Gilchrist KW (1996) Sulcus vocalis: a rational analytical approach to diagnosis and management. Ann Otol Rhinol Laryngol 105(3):189-200. https ://doi.org/10.1177/000348949610500304

3. Bouchayer M, Cornut G, Witzig E, Loire R, Roch JB, Bastian RW (1985) Epidermoid cysts, sulci, and mucosal bridges of the true vocal cord: a report of 157 cases. Laryngoscope 95(9 Pt 1):1087-1094

4. Friedrich G, Dikkers FG, Arens C, Remacle M, Hess M, Giovanni A, Duflo S, Hantzakos A, Bachy V, Gugatschka M (2013) Vocal fold scars: current concepts and future directions. Consensus report of the Phonosurgery Committee of the European Laryngological Society. Eur Arch Oto-rhino-laryngol 270(9):24912507. https://doi.org/10.1007/s00405-013-2498-9

5. Overton L, Adams K, Shah RN, Buckmire RA (2017) Longitudinal voice outcomes after type I Gore-Tex thyroplasty for nonparalytic glottic incompetence. Ann Otol Rhinol Laryngol 126(1):14-19. https://doi.org/10.1177/0003489416672475

6. Jacobson BHJA, Grywalski C, Silbergleit A, Jacobson G, Benninger MS, Newman GW (1997) The Voice Handicap Index (VHI): development and validation. Am J Speech Lang Pathol $6(3): 4$

7. Hakkesteegt MM, Wieringa MH, Gerritsma EJ, Feenstra L (2006) Reproducibility of the Dutch version of the Voice Handicap Index. Folia Phoniatr Logop 58(2):132-138. https://doi. org/10.1159/000089613

8. Van Gogh CD, Mahieu HF, Kuik DJ, Rinkel RN, Langendijk JA, Verdonck-de Leeuw IM (2007) Voice in early glottic cancer compared to benign voice pathology. Eur Arch Otorhinolaryngol 264(9):1033-1038. https://doi.org/10.1007/s00405-007-0313-1

9. Isshiki N, Okamura H, Tanabe M, Morimoto M (1969) Differential diagnosis of hoarseness. Folia Phoniatr 21(1):9-19

10. Beunckens C, Molenberghs G, Kenward MG (2005) Direct likelihood analysis versus simple forms of imputation for missing data in randomized clinical trials. Clin Trials (London, England) 2(5):379-386. https://doi.org/10.1191/1740774505cn119oa

11. Wuyts FL, De Bodt MS, Molenberghs G, Remacle M, Heylen L, Millet B, Van Lierde K, Raes J, Van de Heyning PH (2000) The dysphonia severity index: an objective measure of vocal quality based on a multiparameter approach. J Speech Lang Hear Res JSLHR 43(3):796-809

12. Benninger MS, Hanick AL, Nowacki AS (2016) Augmentation autologous adipose injections in the larynx. Ann Otol Rhinol Laryngol 125(1):25-30. https://doi.org/10.1177/0003489415595427

13. Zeitels SM, Mauri M, Dailey SH (2003) Medialization laryngoplasty with Gore-Tex for voice restoration secondary to glottal incompetence: indications and observations. Ann Otol Rhinol Laryngol 112(2):180-184. https://doi.org/10.1177/0003489403 11200213

14. Hsiung MW, Woo P, Minasian A, Schaefer Mojica J (2000) Fat augmentation for glottic insufficiency. Laryngoscope 110(6):1026-1033. https://doi.org/10.1097/00005537-20000 6000-00026

15. Cantarella G, Mazzola RF, Gaffuri M, Iofrida E, Biondetti P, Forzenigo LV, Pignataro L, Torretta S (2018) Structural fat grafting to improve outcomes of vocal folds' fat augmentation: longterm results. Otolaryngol Head Neck Surg 158(1):135-143. https ://doi.org/10.1177/0194599817739256

16. Lodder WL, Dikkers FG (2015) Comparison of voice outcome after vocal fold augmentation with fat or calcium hydroxylapatite. Laryngoscope 125(5):1161-1165. https://doi.org/10.1002/ lary. 25104

17. Dursun G, Boynukalin S, Ozgursoy OB, Coruh I (2008) Longterm results of different treatment modalities for glottic insufficiency. Am J Otolaryngol 29(1):7-12. https://doi.org/10.1016/j. amjoto.2006.12.001

18. Zelenik K, Walderova R, Kucova H, Jancatova D, Kominek P (2017) Comparison of long-term voice outcomes after vocal fold augmentation using autologous fat injection by direct microlaryngoscopy versus office-based calcium hydroxylapatite injection. Eur Arch Otorhinolaryngol 274(8):3147-3151. https://doi. org/10.1007/s00405-017-4600-1

19. Dominguez ML, Villarreal R, Simpson CB (2018) Voice outcomes of lipoinjection versus medialization laryngoplasty for nonparalytic glottic insufficiency. Laryngoscope 129:1164. https ://doi.org/10.1002/lary.27573

20. Neuenschwander MC, Sataloff RT, Abaza MM, Hawkshaw MJ, Reiter D, Spiegel JR (2001) Management of vocal fold scar with autologous fat implantation: perceptual results. J Voice 15(2):295-304. https://doi.org/10.1016/s0892-1997(01)00031-5 
21. Sanderson JD, Simpson CB (2009) Laryngeal complications after lipoinjection for vocal fold augmentation. Laryngoscope 119(8):1652-1657. https://doi.org/10.1002/lary.20529

22. Chen YY, Pai L, Lin YS, Wang HW, Hsiung MW (2003) Fat augmentation for nonparalytic glottic insufficiency. ORL 65(3):176183. https://doi.org/10.1159/000072256

23. Hsiung MW, Lin YS, Su WF, Wang HW (2003) Autogenous fat injection for vocal fold atrophy. Eur Arch Otorhinolaryngol 260(9):469-474. https://doi.org/10.1007/s00405-003-0622-y

24. Hsiung MW, Chen YY, Pai L, Lin CJ, Wang HW (2003) Results of magnetic resonance imaging assessment, acoustic analysis, phonatory function and perceptual rating of glottic insufficiency before and after fat augmentation: correlated with subjective rating. ORL 65(2):76-83. https://doi.org/10.1159/000070770

25. Stuut M, Tjon Pian Gi RE, Dikkers FG (2014) Change of Voice Handicap Index after treatment of benign laryngeal disorders. Eur Arch Otorhinolaryngol 271(5):1157-1162. https://doi. org/10.1007/s00405-013-2773-9

26. Arffa RE, Krishna P, Gartner-Schmidt J, Rosen CA (2012) Normative values for the Voice Handicap Index-10. J Voice 26(4):462465. https://doi.org/10.1016/j.jvoice.2011.04.006

27. Karle WE, Helman SN, Cooper A, Zhang Y, Pitman MJ (2018) Temporalis fascia transplantation for sulcus vocalis and vocal fold scar: long-term outcomes. Ann Otol Rhinol Laryngol 127(4):223228. https://doi.org/10.1177/0003489417753224

28. Gonzalez-Herranz R, Hernandez Garcia E, Granda-Rosales M, Eisenberg-Plaza G, Montojo Woodeson J, Plaza G (2018) Improved mucosal wave in unilateral autologous temporal fascia graft in sulcus vocalis type 2 and vocal scars. J Voice. https://doi. org/10.1016/j.jvoice.2018.06.013

29. Yilmaz T (2012) Sulcus vocalis: excision, primary suture and medialization laryngoplasty: personal experience with 44 cases. Eur Arch Otorhinolaryngol 269(11):2381-2389. https://doi. org/10.1007/s00405-012-2058-8
30. Miaskiewicz B, Szkielkowska A, Gos E, Panasiewicz A, Wlodarczyk E, Skarzynski PH (2018) Pathological sulcus vocalis: treatment approaches and voice outcomes in 36 patients. Eur Arch Otorhinolaryngol 275(11):2763-2771. https://doi.org/10.1007/ s00405-018-5040-2

31. Hsiung MW, Kang BH, Pai L, Su WF, Lin YH (2004) Combination of fascia transplantation and fat injection into the vocal fold for sulcus vocalis: long-term results. Ann Otol Rhinol Laryngol 113(5):359-366. https://doi.org/10.1177/000348940411300504

32. Hwang CS, Lee HJ, Ha JG, Cho CI, Kim NH, Hong HJ, Choi HS (2013) Use of pulsed dye laser in the treatment of sulcus vocalis. Otolaryngol Head Neck Surg 148(5):804-809. https://doi. org/10.1177/0194599813479909

33. Mortensen MM, Woo P, Ivey C, Thompson C, Carroll L, Altman K (2008) The use of the pulse dye laser in the treatment of vocal fold scar: a preliminary study. Laryngoscope 118(10):1884-1888. https://doi.org/10.1097/MLG.0b013e31817d7546

34. Park YM, Lim JY, Kang MS, Choi HS (2019) Treatment outcomes of angiolytic laser-assisted glottoplasty in patients with sulcus vocalis. Ann Otol Rhinol Laryngol. https://doi.org/10.1177/00034 89419826132

35. Welham NV, Choi SH, Dailey SH, Ford CN, Jiang JJ, Bless DM (2011) Prospective multi-arm evaluation of surgical treatments for vocal fold scar and pathologic sulcus vocalis. Laryngoscope 121(6):1252-1260. https://doi.org/10.1002/lary.21780

Publisher's Note Springer Nature remains neutral with regard to jurisdictional claims in published maps and institutional affiliations. 